\title{
Families of convex sets not representable by points
}

\author{
János Pach* and Géza Tóth ${ }^{\dagger}$ \\ City College of New York, CUNY and \\ Rényi Institute, Hungarian Academy of Sciences
}

\begin{abstract}
Let $(A, B, C)$ be a triple of disjoint closed convex sets in the plane such that each of them contributes at least one point to the boundary $\partial$ of the convex hull of their union. If there are three points $a \in A, b \in B, c \in C$ that belong to $\partial$ and follow each other in clockwise (counterclockwise) order, we say that the orientation of the triple $(A, B, C)$ is clockwise (counterclockwise). We construct families of disjoint closed convex sets $\left\{C_{1}, \ldots, C_{n}\right\}$ in the plane whose every triple has a unique orientation, but there are no points $p_{1}, \ldots, p_{n}$ in general position in the plane whose triples have the same orientations. In other words, these families cannot be represented by a point set of the same order type. This answers a question of A. Hubard and L. Montejano. We also show the size of the largest subfamily representable by points, which can be found in any family of $n$ disjoint closed convex sets in general position in the plane, is $O\left(n^{\log 8 / \log 9}\right)$. Some related Ramsey-type geometric problems are also discussed.
\end{abstract}

\section{Introduction}

Let $\mathcal{C}$ be a family of disjoint closed convex sets in the plane in general position, that is, assume that

1. no three of them have a common tangent line, and

2. the convex hull of the union of two members $A, B \in \mathcal{C}$ never contains a third member $C \in \mathcal{C}$, that is, $\operatorname{conv}(A \cup B \cup C) \neq \operatorname{conv}(A \cup B)$ holds for every triple of distinct members $A, B, C \in \mathcal{C}$.

Analogously, we say that a set of points $P$ in the plane is in general position if no three elements of $P$ are collinear.

\footnotetext{
*Supported by NSF grant CCF-05-14079 and by grants from NSA, PSC-CUNY, BSF, and OTKAK-60427.

${ }^{\dagger}$ Supported by OTKA-K-60427.
} 
The type $\operatorname{tp}(A, B, C)$ of an ordered triple of members of $\mathcal{C}$ is defined as +1 (or -1 ) if there are three points $a \in A, b \in B, c \in C$ belonging to the boundary $\mathrm{Bd} \operatorname{conv}(A \cup B \cup$ $C$ ) that follow each other in clockwise (counterclockwise) order along this boundary. Notice that the same triple $(A, B, C)$ may have two types at the same time if one the sets contributes two arcs to $\operatorname{Bd} \operatorname{conv}(A \cup B \cup C)$.

A point set $P$ in general position in the plane is said to represent the order type of $\mathcal{C}$ if there is a one-to-one correspondence $f: \mathcal{C} \rightarrow P$ such that

$$
\operatorname{tp}(f(A), f(B), f(C))=\operatorname{tp}(A, B, C) \text { for all } A, B, C \in \mathcal{C} \text { with a unique type. }
$$

As far as we know, order types of set families were first studied by Bisztriczky and G. Fejes Tóth [BF1],[BF2]. They generalized a famous conjecture of Erdős and Szekeres [ES1],[ES2] to families of convex sets in the plane as follows: Any family $\mathcal{C}$ of at least $2^{n-2}+1$ disjoint closed convex sets in general position has $n$ members in convex position. Recently, A. Hubard and L. Montejano suggested that this stronger conjecture may actually be equivalent to the original one. More precisely, they suspected that the order type of every family $\mathcal{C}$ with the above property can be represented by points. In the present note, we show that this is not the case.

Theorem 1. There exists a family of nine pairwise disjoint segments in general position in the plane, whose order type cannot be represented by points.

Let $r=r(n)$ denote the largest integer such that every family $\mathcal{C}$ of $n$ disjoint closed convex sets in general position in the plane has $r$ members whose order type can be represented by points. By definition, the order type of any subfamily of $\mathcal{C}$ in convex position can be represented by points. According to [PT1], every family $\mathcal{C}$ with the above property has at least $\log _{16} n$ members in convex position. Therefore, we have $r(n) \geq \log _{16} n$. Iterating the construction in Theorem 1, we obtain

Theorem 2. For every $n$, there exists a family of $n$ pairwise disjoint segments in general position in the plane which has no subfamily of size $\left\lfloor n^{\log 8 / \log 9}\right\rfloor$ whose order type is representable by points.

A collection of two-way infinite (unbounded) non-selfintersecting curves in the plane is called a family of pseudolines if any two curves have precisely one point in common, at which they properly cross. It is said to be simple if no three pseudolines pass through the same point. A family of pseudolines $\mathcal{P}$ is stretchable if there exists a family of lines $\mathcal{L}$ such that the cell decompositions induced by $\mathcal{P}$ and $\mathcal{L}$ are topologically isomorphic. It was known already to Hilbert $[\mathrm{H}]$ and Levi $[\mathrm{L}]$ that there are nonstretchable families of pseudolines. The first example of a nonstretchable simple arrangement was given by Ringel [R]. It was shown by Mnev [M1, M2] that it is a computationally hard ( $N P$-hard) problem to decide whether an arrangement of pseudolines is stretchable (see also Shor $[\mathrm{S}]$.)

In complete analogy to the above problem, we can try to determine the size of the largest stretchable subfamily contained in every simple family of $n$ pseudolines. 
Theorem 3. Let $s=s(n)$ denote the largest integer such that every simple family $\mathcal{P}$ of $n$ pseudolines has a stretchable subfamily of size s. For every n, we have

$$
\log _{4} n \leq s(n) \leq\left\lfloor n^{\log 8 / \log 9}\right\rfloor .
$$

Theorem 1 is established in Section 2, Theorems 2 and 3 are proved in Section 3. In the last section, we discuss some related problems.

\section{A nonrepresentable order type of segments}

The aim of this section is to establish Theorem 1. The proof is based on Ringel's $[\mathrm{R}]$ construction of a nonstretchable arrangement of nine pseudolines, that can be obtained by modifying the Pappus configuration; see Figure 1. It is known that every arrangement of fewer than nine pseudolines is stretchable [GP1], [GP3].

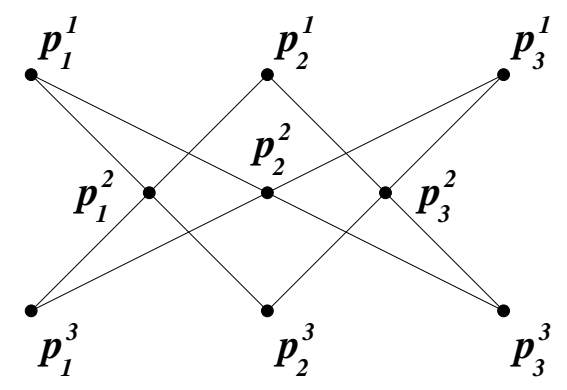

Figure 1: The Pappus configuration.

Let $\mathcal{S}=\left\{S_{1}, \ldots, S_{n}\right\}$ be a family of disjoint segments in general position in the plane. We say that $\mathcal{S}$ can be flattened if for any $\varepsilon>0$ there are two disks of radius $\varepsilon$ at unit distance, $D_{1}$ and $D_{2}$, and another family of disjoint segments $\mathcal{S}^{\prime}=\left\{S_{1}^{\prime}, \ldots, S_{n}^{\prime}\right\}$ with the same order type such that each $S_{i}^{\prime} \in \mathcal{S}^{\prime}$ has one endpoint in $D_{1}$ and one in $D_{2}$.

Lemma 1. There exists a family $\mathcal{S}$ of nine segments in general position in the plane

(i) which can be flattened, and

(ii) the order type of which cannot be represented by points.

Proof. Start with the Pappus configuration (see Figure 1), and slightly perturb its points so that its originally collinear triples receive the following orientations:

$$
\operatorname{tp}\left(p_{1}^{1}, p_{2}^{1}, p_{3}^{1}\right)=+1, \quad \operatorname{tp}\left(p_{1}^{2}, p_{2}^{2}, p_{3}^{2}\right)=+1, \quad \operatorname{tp}\left(p_{1}^{3}, p_{2}^{3}, p_{3}^{3}\right)=+1,
$$




$$
\begin{array}{ll}
\operatorname{tp}\left(p_{1}^{1}, p_{1}^{2}, p_{2}^{3}\right)=+1, & \operatorname{tp}\left(p_{1}^{1}, p_{2}^{2}, p_{3}^{3}\right)=-1, \\
\operatorname{tp}\left(p_{2}^{1}, p_{1}^{2}, p_{1}^{3}\right)=-1, & \operatorname{tp}\left(p_{2}^{1}, p_{3}^{2}, p_{3}^{3}\right)=+1, \\
\operatorname{tp}\left(p_{3}^{1}, p_{3}^{2}, p_{2}^{3}\right)=-1, \quad \operatorname{tp}\left(p_{3}^{1}, p_{2}^{2}, p_{1}^{3}\right)=-1 .
\end{array}
$$

This can be achieved, for example, by taking

$$
\begin{gathered}
p_{1}^{1}=(-2,1), \quad p_{2}^{1}=(0,1+110 \delta), \quad p_{3}^{1}=(2,1), \\
p_{1}^{2}=(-1,-2 \delta), \quad p_{2}^{2}=(0,-\delta), \quad p_{3}^{2}=(1,-2 \delta), \\
p_{1}^{1}=(-2,-1-50 \delta), \quad p_{2}^{3}=(0,-1-20 \delta), \quad p_{3}^{3}=(2,-1) .
\end{gathered}
$$

It follows from Ringel's result $[\mathrm{R}]$, by duality, that there are no points $\bar{p}_{j}^{i}(1 \leq$ $i, j \leq 3$ ), for which the above nine triples have the same types (orientations) as for the points $p_{j}^{i}(1 \leq i, j \leq 3)$, except that the orientation of the last triple is opposite, that is, $\operatorname{tp}\left(\bar{p}_{3}^{1}, \bar{p}_{2}^{2}, \bar{p}_{1}^{3}\right)=+1$. In other words, this modified order type $\tau$ is not representable by points (see Figure 2).

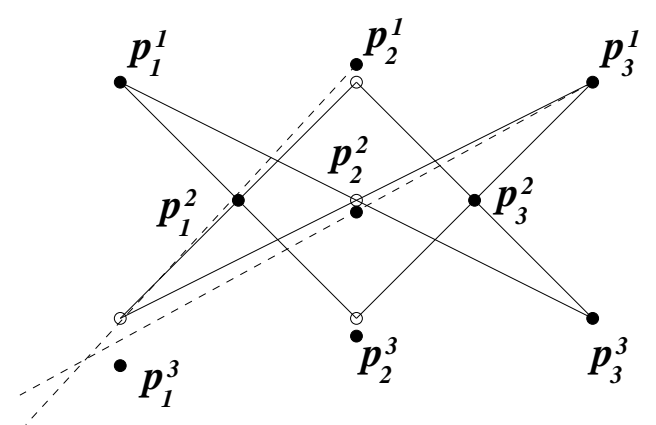

Figure 2: The set $\left\{p_{j}^{i} \mid 1 \leq i, j \leq 3\right\}$.

Next we show that there is a family of segments whose order type is $\tau$.

Let $L$ be a fixed very large number. For any $1 \leq i, j \leq 3$, let $S_{j}^{i}$ be a segment of length $L$ with slope $1 / 2$, whose right endpoint is $p_{j}^{i}$. Observe that the set of segments $\left\{S_{j}^{i} \mid 1 \leq i, j \leq 3\right\}$ has the same order type as the point set $\left\{p_{j}^{i} \mid 1 \leq i, j \leq 3\right\}$. Now slightly rotate $S_{3}^{1}$ about $p_{3}^{1}$ in the clockwise direction to a position in which $p_{2}^{2}, p_{1}^{3}$, and hence the entire segment $S_{1}^{3}$, lie below it. Next, applying a small counterclockwise rotation about $p_{2}^{2}$, bring the segment $S_{2}^{2}$ into a position where it lies below $S_{1}^{3}$. See Figure 3. With a slight abuse of notation, the new segments are also denoted by $S_{j}^{i}$. Notice that during the above transformation no triple of segments $S_{j}^{i}$ switched 
orientations, except one: the orientation of $\left(S_{3}^{1}, S_{2}^{2}, S_{1}^{3}\right)$ has become clockwise. That is, we have

$$
\begin{gathered}
\operatorname{tp}\left(S_{1}^{1}, S_{2}^{1}, S_{3}^{1}\right)=+1, \quad \operatorname{tp}\left(S_{1}^{2}, S_{2}^{2}, S_{3}^{2}\right)=+1, \quad \operatorname{tp}\left(S_{1}^{3} S_{2}^{3} S_{3}^{3}\right)=+1, \\
\operatorname{tp}\left(S_{1}^{1}, S_{1}^{2}, S_{2}^{3}\right)=+1, \quad \operatorname{tp}\left(S_{1}^{1}, S_{2}^{2}, S_{3}^{3}\right)=-1 \\
\operatorname{tp}\left(S_{2}^{1}, S_{1}^{2}, S_{1}^{3}\right)=-1, \quad \operatorname{tp}\left(S_{2}^{1}, S_{3}^{2}, S_{3}^{3}\right)=+1 \\
\operatorname{tp}\left(S_{3}^{1}, S_{3}^{2}, S_{2}^{3}\right)=-1, \quad \operatorname{tp}\left(S_{3}^{1}, S_{2}^{2}, S_{1}^{3}\right)=+1 .
\end{gathered}
$$

Consequently, we found a family of segments whose order type $\tau$ is not representable by points.

It remains to argue that the family $\mathcal{S}=\left\{S_{j}^{i} \mid 1 \leq i, j \leq 3\right\}$ can be flattened. To see this, notice that for any $\varepsilon>0$, one can choose a sufficiently small $\delta$ and a sufficiently large $L$ so that, after appropriate scaling, all left endpoints and all right endpoints of the segments lie in two disks of radius $\varepsilon$ at unit distance from each other.

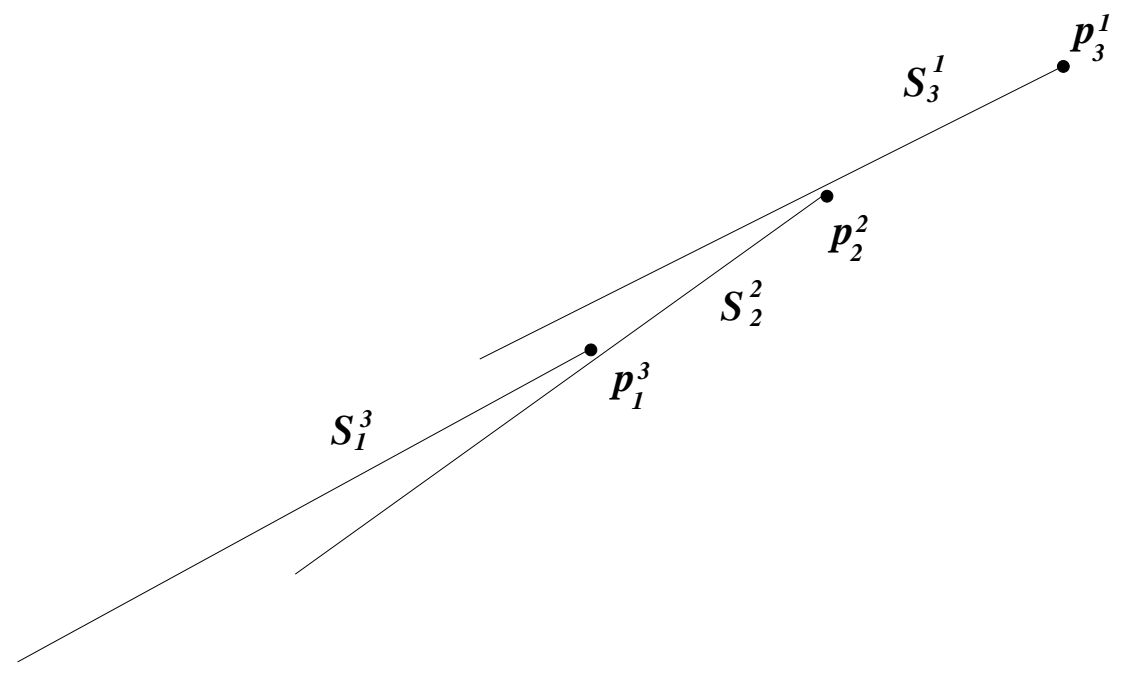

Figure 3: The triple $\left(S_{3}^{1}, S_{2}^{2}, S_{1}^{3}\right)$ switched its orientation.

\section{The iteration step}

Theorem 2 is an immediate corollary of Lemma 1 and the following statement. 
Lemma 2. Suppose there exists a family $\mathcal{S}$ of $k$ disjoint segments in general position in the plane, which can be flattened and which has an order type that cannot be represented by points. Then, for every $i=1,2, \ldots$, there is a family $\mathcal{S}^{i}$ of $k^{i}$ segments in general position in the plane, which can be flattened and which does not have any subfamily of size larger than $(k-1)^{i}$ whose order type can be represented by points.

Proof. For any $\delta>0$, let $\mathcal{S}^{1}(\delta)$ be a family of $k$ unit segments whose order type cannot be represented by points and their left endpoints and right endpoints lie in two disks of radius $\delta$. Let $i \geq 1$ and suppose, recursively, that we have already constructed a family $\mathcal{S}^{i}=\left\{S_{1}, \ldots, S_{k^{i}}\right\}$ of unit segments such that their left and right endpoints lie in two disks of radius $\varepsilon / 2$ and $\mathcal{S}^{i}$ has no subfamily of size larger than $(k-1)^{i}$, whose order type can be represented by points.

Let $\delta$ be a small positive number to be specified later. Replace each segment $S_{j} \in \mathcal{S}^{i}$ by a congruent copy $\mathcal{S}_{j}^{1}(\delta)$ of $\mathcal{S}^{1}(\delta)$, with the same orientation, in such a way that $S_{j}$ coincides with a member of $\mathcal{S}_{j}^{1}(\delta)$. Let $\mathcal{S}^{i+1}$ be the union of these copies. Obviously, we have $\left|\mathcal{S}^{i+1}\right|=k^{i+1}$. Furthermore, if $\delta>0$ is sufficiently small, then

1. the members of $\mathcal{S}^{i+1}$ are disjoint and are in general position;

2. the left endpoints and the right endpoints of the segments in $\mathcal{S}^{i+1}$ lie in two disks of radius at most $\varepsilon / 2+\delta \leq \varepsilon$;

3. for any three distinct indices $j, j^{\prime}, j^{\prime \prime}$, the orientation of any triple of segments belonging to the subfamilies replacing $S_{j}, S_{j^{\prime}}, S_{j^{\prime \prime}}$, respectively, is the same as the orientation of the triple $\left(S_{j}, S_{j^{\prime}}, S_{j^{\prime \prime}}\right)$.

It is easy to show that one cannot select more than $(k-1)^{i+1}$ segments from $\mathcal{S}^{i+1}$ such that their order type can be represented by points. Indeed, by property 3 above and by the induction hypothesis, such a set cannot contain segments belonging to more than $(k-1)^{i}$ subfamilies $\mathcal{S}_{j}^{1}(\delta)$ replacing distinct elements $S_{j} \in \mathcal{S}^{i}$. On the other hand, from each subfamily $\mathcal{S}_{j}^{1}(\delta)$, we can select at most $k-1$ segments.

In the same way, as Theorem 2 can be deduced from Theorem 1 using Lemma 2, one can establish the upper bound in Theorem 3 by iterating Ringel's construction of a nonstretchable arrangement of nine pseudolines $[R]$. By the result of Goodman $[\mathrm{G}]$ every arrangement of pseudolines can be represented such that all pseudolines are $x$-monotone curves, in particular, it follows that Ringel's construction can be "flattened" in the following sense: An arrangement of pseudolines $\mathcal{P}=\left\{\pi_{i} \mid i \in I\right\}$ can be flattened if for any $\varepsilon>0$, there exist real functions $f_{i}: \mathbf{R} \rightarrow \mathbf{R}, i \in I$ satisfying two conditions.

1. The graphs of the functions $f_{i}$ form an arrangement of pseudolines such that the cell decomposition of the plane induced by them is isomorphic to the cell decomposition induced by $\mathcal{P}$. 
2. For every $i \in I$ and $x \in \mathbf{R}$, we have $\left|f_{i}(x)\right|<\varepsilon$.

To prove the upper bound in Theorem 3, instead of Lemma 2 we have to use the following statement (the straightforward recursive proof of which is left to the reader).

Lemma 3. Suppose there exists a simple nonstretchable arrangement $\mathcal{P}$ of $k$ pseudolines in the plane, which can be flattened. Then, for every $i=1,2, \ldots$, there is a simple nonstretchable arrangement $\mathcal{P}^{i}$ of $k^{i}$ pseudolines which can be flattened and which does not have any stretchable subarrangement of size larger than $(k-1)^{i}$.

It was first pointed out by Goodman and Pollack [GP2] that every finite arrangement of pseudolines is isomorphic to an arrangement of $x$-monotone pseudolines (see also [GP3] and [GPWZ] for a much stronger statement). Therefore, to prove the lower bound in Theorem 3, it is enough to restrict our attention to families of $x$-monotone pseudolines.

Let $\mathcal{P}=\left\{\ell_{1}, \ell_{2}, \ldots, \ell_{n}\right\}$ be a simple arrangement of $x$-monotone pseudolines. We say that $\ell_{1}, \ell_{2}, \ldots, \ell_{n}$ form a cap (a cup) if

1. in the cell decomposition determined by them there is an unbounded cell whose boundary contains a piece of each member of $\mathcal{P}$, in this clockwise (counterclockwise) order, and

2. this cell lies below (above) every pseudoline $\ell_{i} \in \mathcal{P}$.

It is clear that all caps and cups $\mathcal{P}$ are stretchable, since the cell decomposition of the plane induced by them is isomorphic to the cell decomposition induced by $|\mathcal{P}|$ distinct tangent lines of an open semicircle.

Thus, the lower bound in Theorem 3 follows from

Lemma 4. Any simple arrangement $\mathcal{P}$ of more than $\left(\begin{array}{c}k+m-4 \\ m-2\end{array}\right) x$-monotone pseudolines contains a cap of size at least $k$ or a cup of size at least $m$.

Proof. The statement can be established by dualizing and adapting the original proof of the Erdös-Szekeres theorem [ES1]. The lemma holds if $k \leq 2$ or $m \leq 2$. Suppose that $k, m>2$ are fixed and that we have already proved the statement for all pairs $\left(k^{\prime}, m^{\prime}\right)$ with $k^{\prime}<k$ or $m^{\prime}<m$.

Let $\mathcal{P}$ be a simple arrangement of $\left(\begin{array}{c}k+m-4 \\ m-2\end{array}\right)+1 x$-monotone pseudolines. Using the induction hypothesis and the fact that $\left(\begin{array}{c}k+m-4 \\ m-2\end{array}\right)+1>\left(\begin{array}{c}k+m-5 \\ m-2\end{array}\right)+1$, we obtain that $\mathcal{P}$ contains a cap of size $(k-1)$ or a cup of size $m$. In the latter case we are done. So we may assume that $\mathcal{P}$ contains a cap of size $(k-1)$. Delete the first member of such a cap from the arrangement. We still have more than $\left(\begin{array}{c}k+m-5 \\ m-2\end{array}\right)+1$ pseudolines, so $\mathcal{P}$ must have another cap of size $(k-1)$. Again, delete its first member, and repeat this procedure as long as there are more than $\left(\begin{array}{c}k+m-5 \\ m-2\end{array}\right)$ pseudolines left. Then stop. We have deleted altogether $\left(\begin{array}{c}k+m-4 \\ m-2\end{array}\right)+1-\left(\begin{array}{c}k+m-5 \\ m-2\end{array}\right)=\left(\begin{array}{c}k+m-5 \\ m-3\end{array}\right)+1$ pseudolines. Therefore, by the induction hypothesis, the set of deleted pseudolines must contain a cap of size 
$k$ or a cup of size $(m-1)$. In the first case, we are done. In the second case, there exists a cup $\mathcal{C}_{1}$ of size $(m-1)$ such that its first member is also the first member of a cap $\mathcal{C}_{2}$ of size $(k-1)$. It is easy to verify (see Figure 4 ) that

1. either one can extend $\mathcal{C}_{1}$ by the second member of $\mathcal{C}_{2}$ to a cup of size $m$,

2. or one can extend $\mathcal{C}_{2}$ by the second member of $\mathcal{C}_{1}$ to a cap of size $k$.

This completes the proof.

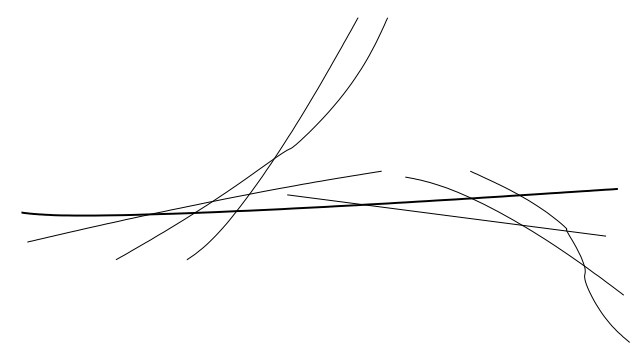

Figure 4: A cap and a cup of size four, having the same first member.

\section{Concluding remarks}

The lower bounds for the functions $r(n)$ and $s(n)$ (in Theorem 3) follow from ErdösSzekeres type results: from the existence of large convex subconfigurations. To further improve these bounds, we need to find larger classes $\Gamma$ of "unavoidable" configurations, representable by points, such that for any $k$, every sufficiently large system contains a subconfiguration of size $k$ belonging to $\Gamma$. To improve the upper bounds, on the other hand, we have to define more complicated operations for building large nonrepresentable order types or nonstretchable arrangements of pseudolines, using smaller examples.

One can extend the definition of order type to arbitrary families of disjoint convex sets, no three of which have a common tangent line, as follows. Suppose that a member of $\mathcal{C}$ is allowed to lie "between" two other members, that is, it can be contained in the convex hull of the union of two others. If, for example, $B \subset \operatorname{conv}(A \cup C)$ for some $A, B, C \in \mathcal{C}$, let $\operatorname{tp}(A, B, C)$ and the type of every permutation of these three members be zero. In all other cases, let us define $\operatorname{tp}(A, B, C)$ as before.

We can now say that a point set $P$ in general position in the plane represents the order type of $\mathcal{C}$ if there is a one-to-one correspondence $f: \mathcal{C} \rightarrow P$ such that

$\operatorname{tp}(f(A), f(B), f(C))=\operatorname{tp}(A, B, C)$ for all $A, B, C \in \mathcal{C}$ with a unique nonzero type. 
Note that, according to this definition, any set of $n$ points in general position represents the order type of a family of disjoint convex bodies lying between and touching the graphs of two functions $f, g: \mathbf{R} \rightarrow \mathbf{R}$, where $f$ is strictly concave, $g$ is strictly convex, and $f(x) \leq g(x)$ for every $x \in \mathbf{R}$.

By Ramsey's theorem for three-uniform hypergraphs, every family $\mathcal{C}$ of $n$ disjoint convex sets in the plane contains a large (that is, roughly $\log \log n$ size) subfamily $\mathcal{C}^{\prime} \subseteq \mathcal{C}$, in which either the type of every triple is zero or the type of no triple is zero [GRS]. In the former case, the order type $\mathcal{C}^{\prime}$ can be represented by any set of points in general position, in the latter one, we can apply the results of [PT1] to argue that $\mathcal{C}^{\prime}$ has a large subfamily, the order type of which can be represented by points.

\section{References}

[BF1] T. Bisztriczky and G. Fejes Tóth, A generalization of the Erdős-Szekeres convex n-gon theorem, Journal für die reine und angewandte Mathematik 395 (1989), $167-170$.

[BF2] T. Bisztriczky and G. Fejes Tóth, Convexly independent sets, Combinatorica 10 (1990), 195-202.

[ES1] P. Erdős and G. Szekeres, A combinatorial problem in geometry, Compositio Mathematica 2 (1935), 463-470.

[ES2] P. Erdős and G. Szekeres, On some extremum problems in elementary geometry, Ann. Universitatis Scientiarum Budapestinensis, Eötvös, Sectio Mathematica III-IV (1960-61), 53-62.

[F] G. Fejes Tóth, Recent progress on packing and covering, in: Advances in Discrete and Computational Geometry (B. Chazelle et al., eds.), Contemporary Mathematics, Vol. 223, AMS, Providence, 1999.

[G] J. E. Goodman, Proof of a conjecture of Burr, Grünbaum, and Sloane, Discrete Mathematics 32 (1980), 27-35.

[GP1] J. E. Goodman and R. Pollack, Proof of Grünbaum's conjecture on the stretchability of certain arrangements of pseudolines, J. Comb. Theory, Ser. A 29 (1980), 385-390.

[GP2] J. E. Goodman and R. Pollack, Semispaces of configurations, cell complexes of arrangements, J. Comb. Theory, Ser. A 37 (1984), 257-293.

[GP3] J. E. Goodman and R. Pollack, Allowable sequences and order types in discrete and computational geometry, in: New Trends in Discrete and Computational Geometry (J. Pach, ed.), Algorithms and Combinatorics 10, Springer, Berlin, 1993, 103-134. 
[GPWZ] J. E. Goodman, R. Pollack, R. Wenger, and T. Zamfirescu, Arrangements and topological planes, Amer. Math. Monthly 101 (1994), 866-878.

[GRS] R.L. Graham, B.L. Rothschild, and J.H. Spencer, Ramsey Theory, 2nd ed., John Wiley, New York, 1990.

[H] D. Hilbert, The Foundations of Geometry, 2nd ed., Open Court, Chicago, 1910.

[L] F. Levi, Die Teilung der projektiven Ebene durch Gerade oder Pseudogerade, Ber. Math.-Phys. Kl. sächs. Akad. Wiss. Leipzig, 78 (1926), 256-267.

[M1] N. E. Mnev, Varieties of combinatorial types of projective configurations and convex polyhedra (in Russian), Dokl. Akad. Nauk SSSR 283 (1985), 1312-1314.

[M2] N. E. Mnev, The universality theorems on the classification problem of configuration varieties and convex polytopes varieties, in: Topology and geometryRohlin Seminar, Lecture Notes in Mathematics 1346, Springer, Berlin, 1988, $527-543$.

[PT1] J. Pach and G. Tóth, A generalization of the Erdős-Szekeres theorem to disjoint convex sets, Discrete and Computational Geometry 19 (1998), 437-445.

[PT2] J. Pach and G. Tóth, Erdös-Szekeres-type theorems for segments and noncrossing convex sets, Geometriae Dedicata 81 (2000), 1-12.

[R] G. Ringel, Teilungen der projectiven Ebene durch Geraden oder topologische Geraden, Math. Z. 64 (1956), 79-102.

[S] P. W. Shor, Stretchability of pseudolines is NP-hard, in: Applied Geometry and Discrete Mathematics, DIMACS Ser. Discrete Math. Theoret. Comput. Sci. 4, Amer. Math. Soc., Providence, RI, 1991, 531-554. 